\title{
Managing essential healthcare services during the first nationwide lockdown: experiences from a Regional Referral Hospital in Bhutan
}

Purushotam Bhandari ${ }^{1}$, Neelima Chhetri², Dinesh Pradhan ${ }^{3}$

${ }^{1,3}$ Department of Pediatrics, Central Regional Referral Hospital, Gelephu, Bhutan

${ }^{2}$ Department of Surgery, Central Regional Referral Hospital, Gelephu, Bhutan

\begin{abstract}
The current Coronavirus Disease of 2019 (COVID-19) pandemic has caused unprecedented burden on healthcare systems, derailing some of the most efficient and established health systems in the world. As the pandemic has diverted all attention and priorities of health care services, there is a significant concern that non-COVID essential healthcare services may receive less attention. Lockdowns have become an important public health measure to contain local outbreaks of COVID-19. We describe the experiences of the Central Regional Referral Hospital, Gelephu, in ensuring the continuity of provision of essential healthcare services during the first nationwide lockdown in August-September, 2020. Adequate preparations with a well-thought of contingency plan and identification of roles is vital. A good communication system backed by a 24-hour hotline is an essential component. A mobile clinic with the most essential services like mother and child health $(\mathrm{MCH})$ services, immunizations, medicine refills and non communicable disease care, form important components of essential healthcare services. We believe that the description of our experience will be helpful for other hospitals in the country to optimize management of essential services during the future lockdowns.
\end{abstract}

Keywords: Essential healthcare services; Lockdown; Mobile clinic.

\section{INTRODUCTION}

The COVID-19 pandemic has caused an unprecedented burden on healthcare services throughout the world. Being a neighbor of one of the worst affected countries in the world, Bhutan recognized the risk early on in the pandemic and made excellent efforts towards curtailing the virus. Bhutan reported the first case of COVID-19 in March 2020 in an American tourist ${ }^{1}$. As of October 2021, Bhutan has reported over 2600 cases and 3 deaths due to COVID-192. Bhutan enforced its first nationwide lockdown in August-September 2020 following the detection of a community case in Gelephu on 10th August, 2020 followed by a larger community outbreak in Phuntsholing on $12^{\text {th }}$ August, 2020.

In a disease outbreak situation, lockdown becomes an inevitable measure to contain further spread of disease and buy time for surveillance and case finding. Lockdown is one of the most effective non-pharmacological interventions in controlling an epidemic or a pandemic ${ }^{3}$. However, it becomes a major challenge for the health sector to deliver the routine essential healthcare services during lockdowns. In order to prevent illnesses and deaths from non- COVID conditions, essential healthcare services must be ensured continuously during a lockdown ${ }^{4}$.

This paper presents the experiences of Central Regional Referral Hospital, Gelephu, in managing the provision of essential healthcare services during the first nationwide lockdown.

\section{Definition of Essential Health Services}

An Essential Health Package in a low-income country consists of a limited list of public health and clinical services which will be provided at primary and/or secondary care level ${ }^{5}$. The components of the package vary from country to country depending on their level of development and priorities. While they are routinely met at normal times, the provision of essential healthcare services becomes challenging during a pandemic, especially during lockdowns.

For the COVID-19 pandemic, Bhutan's Ministry of Health identified its own list of Essential Services to be provided in the event of a lockdown ${ }^{6}$. Four broad areas had been identified which included child immunization, mother and child health services, medicine refills and chronic health conditions. The Ministry of Health also prepared a contingency plan for managing essential healthcare services during lockdowns, which served as a guideline for healthcare facilities. 


\section{What were the preparations?}

Being situated on the border with India, Gelephu had always been on high alert for imported cases of COVID-19 and a local outbreak. Therefore, a contingency plan in line with the $\mathrm{MoH}$ guideline was developed for delivering the most essential healthcare services during a lockdown. A designated focal person for essential services was identified along with a separate list of heath staff to provide different categories of services. A 24 hourhotline manned by a senior specialist and a social media group of focal persons was established for wider communications. Color printed pamphlets with the details of services provided, names of focal persons for various services and their contact details were printed and kept ready. A school bus was also identified for the mobile clinic services.

\section{How did we begin?}

As soon as the news of the first nationwide lockdown was announced on $10^{\text {th }}$ August 2020, the Essential Services Team was activated at $4 \mathrm{~A} . \mathrm{M}$ in the morning followed by a brief coordination meeting at 8 A.M on $11^{\text {th }}$ August. By 9 A.M, Mobile Clinic Team was already in the field along with all identified services. By 12 noon, pamphlet distribution throughout our catchment areas was completed with the help of the local Tshogpas (village representatives).We were also able to disseminate necessary information and contact numbers in the local Television channel and social media groups by noon time on the same day.

\section{Major components of our essential healthcare services:}

- 24 x 7 hotline manned by a senior specialist medical doctor.

- Mobile clinic with the most essential services like mother and child health, immunization, medicine refill and chronic disease follow up.

- One Mini Mobile Clinic for more urgent cases.

- Medicine drops services at doorsteps

- 24- hour Emergency Room(existing)

- Normal inpatient care services

- Uninterrupted hemo-dialysis services

\section{The hotline}

During the entire lockdown which lasted for 37 days in Gelephu, the hotline received approximately 2000 calls averaging 50 calls per day. A breakdown of 440 hotline calls received in the first ten days of the lockdown is presented in Figure 1. Majority of the calls on the hotline were for consultations of minor illnesses like fever and cough, followed by medicine refill and $\mathrm{MCH}$ related services.

\section{Mobile Clinics: the backbone of Essential Health Services}

During the lockdown, hospital's outpatient services were suspended, with most of the non-emergency services being delivered by a Mobile Clinic manned by staff from MCH clinic, non-communicable diseases (NCD) clinic, pharmacy, laboratory, and clinical services. The following services were rendered at the community level through the mobile clinics.
- Normal outpatient services

- $\mathrm{MCH}$ services

- Non--Communicable Diseases

- Medicine refills

- $\quad$ First aid

- Minor emergencies

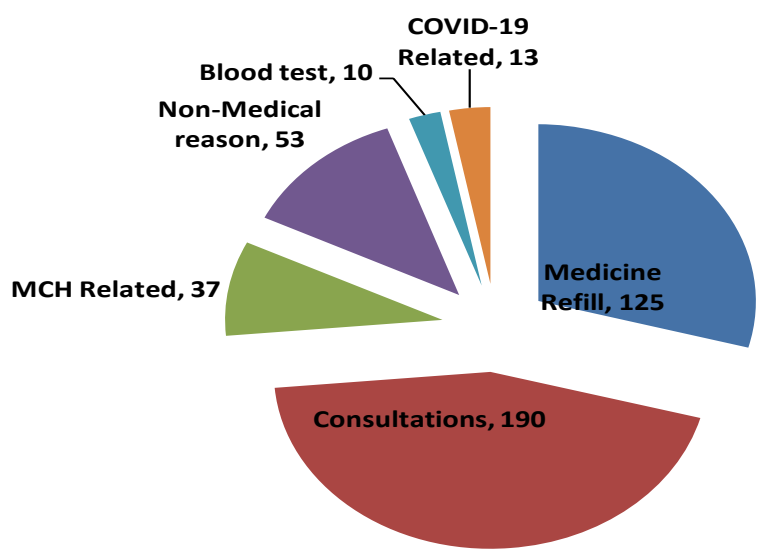

Figure 1. Breakdown of 440 hotline calls for the first ten days of lockdown

Major Service beneficiaries: NCDs

A total of 3372 clients were seen by the Mobile clinic during the entire period of the first nationwide lockdown that lasted for a total of 37 days. It was observed that more than half of the clients availing the services were either having diabetes, hypertension or both.

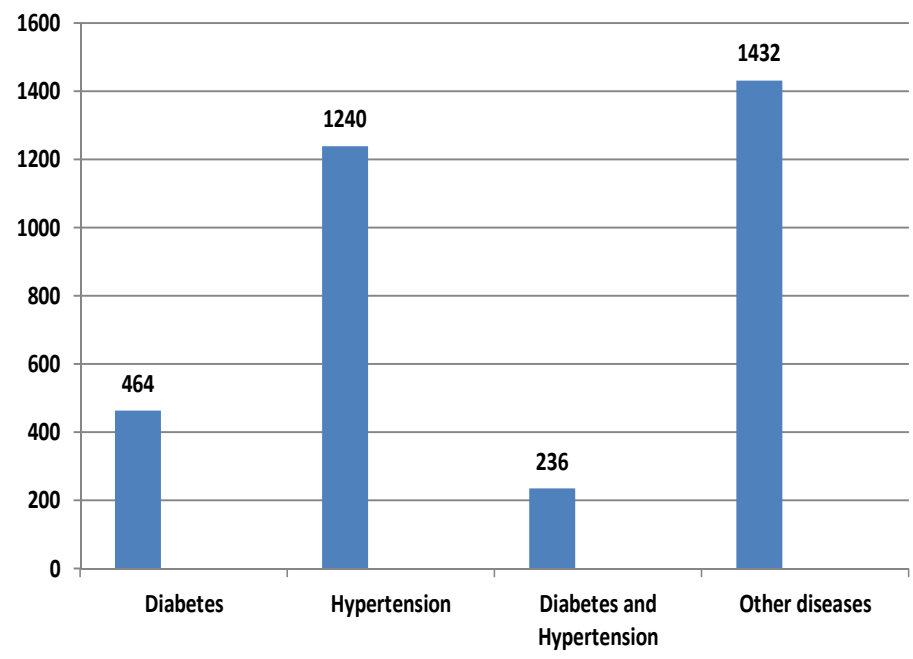

Figure 2. Chart showing the proportion of clients with NCDs

\section{MCH services}

Family planning was the most common service sought in the mobile clinic followed by ante-natal follow up and de-worming using Albendazole and vitamin A supplementation. Although weighing of children was initially not planned in the essential healthcare services, it was later included due to public demand. 


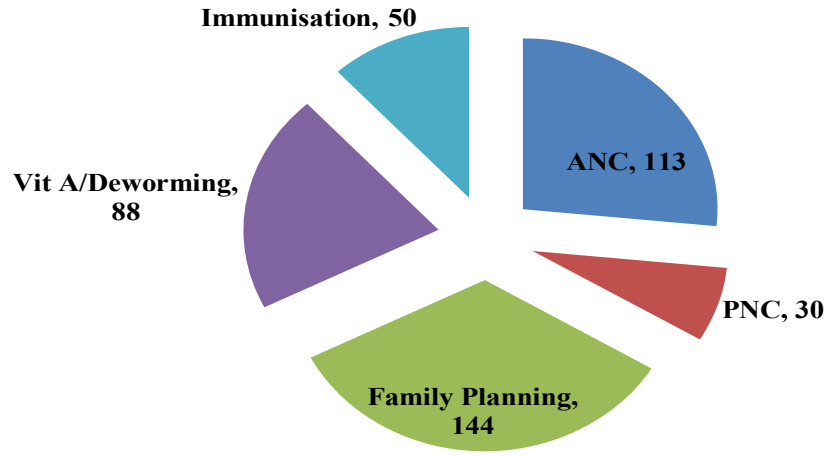

Figure 3. Breakdown of MCH services availed in the Mobile Clinics

\section{Medicine Refills}

After medical consultation, the second most common reason for the hotline call was to avail medicine refills. Refills for noncommunicable diseases like diabetes and hypertension or both was the most common amongst the medicine refills. Following a client's call, a hand-written prescription was made by the senior specialist doctor handling the hotline and passed on to the pharmacy team. Volunteers amongst the hospital staff and Bhutan Red Cross Society (BRCS) came forward voluntarily during the lockdown and dropped the medicines to the clients' houses in their personal cars. However, majority of medicine refills were handled by the Mobile Clinics.

\section{Dialysis Services}

Chronic kidney disease patients received un-interrupted hemodialysis services during the lockdown. An average of ten dialysis sessions was conducted daily. A separate movement permit was issued to those patients who had personal cars to facilitate smooth travel to and from the hospital. Throughout the lockdown, BRCS volunteers played an important role in providing free pickup and drop services to patients who did not have personal cars.

\section{Pickup and Drop services}

Hospital ambulances were deputed to pick up serious and emergency cases to the hospital. In certain situations, patients were allowed to travel to hospital in their personal cars, especially when the ambulances were fully engaged. Patients discharged from the hospital were dropped home by the BRCS volunteers who were stationed at the hospital's lobby throughout the lockdown.

\section{In-patient services}

All admissions were channeled through the hospital's 24-hour emergency room and all inpatient services were provided as usual, without any hindrance. While elective surgeries were postponed, emergency surgeries and procedures were performed un-interrupted. A random check on inpatient statistics on one of the days of the lockdown revealed that the Emergency room, Pediatric, Maternity and Medical wards were the busiest units with maximum patient turn over even during the lockdown. This is the usual trend during normal times and does not necessarily mean an increased workload during the lockdown.

\section{Communication channels}

Apart from a 24-hour hotline which served as an effective telephonic triage, other communication channels were opened and accessible to the general public. Regular updates in social media and local television channels provided additional modes of disseminating information. Social messaging apps proved useful in receiving patient prescriptions and distribution of the medicines in addition to being an important channel of communications amongst service providers. Apart from providing health-related information and advice, information related to other essential services like groceries and vegetables were also provided occasionally.

\section{Issues and challenges}

With the contingency plan and designated staff in place, the hospital was able to cater to the essential health care services smoothly during the entire lockdown. There were no unexpected deaths or major delays in providing critical services to the community. However, some of the major challenges faced during the provision of essential healthcare services during the lockdown were:

- Movement of health staff to-and-from hospital, since vehicle movements were restricted even for health workers.

- Hospital ambulances and utility vehicles were overwhelmed in the initial few days.

- Patients not being able to identify the names and dosages of their regular medicines.

- Mobile Clinics having to face the scorching heat and the heavy monsoon rains.

- Long queues in mobile clinic

- Public demand for non-essential services like weighing their child and vitamin supplements, even during lockdowns.

- Shortage of clinicians for the Mobile Clinic, as they had to be mobilized from the available hospital pool of doctors and clinicians.

\section{RECOMMENDATIONS}

The entire experience of providing essential healthcare services during a lockdown lasting more than five weeks was a unique learning opportunity for the authorities and staff of the Regional Referral Hospital. With the pandemic showing no signs of abating and mutant variants being increasingly identified, future lockdowns are inevitable. The following measures we adopted helped us in planning and providing efficient essential healthcare services during such situations and may be useful for other health centers in the country.

- Prepare a comprehensive Hospital Contingency Plan with a focal person exclusively dedicated for essential healthcare services. 
- Establish effective communication channels including hotline to effectively triage phone calls and provide essential information.

- Define clear roles and responsibilities of personnel providing essential healthcare services.

- Focus on NCDs, medicine refills and out-patient services in the community. A detailed list of people with NCDs, differentially-abled, immunocompromised and special care needs, should be prepared in advance.

- Ensure smooth and hassle-free movement of health staff with vehicle movement stickers.

- Ensure adequate vehicles for patient pick up and drop services.

- Sensitize public to keep the most essential over-the-counter drugs at home (Paracetamol, antacids, ORS, antiseptic soap, band aids).

- Identify social media platform to receive patient prescriptions for medicine refills.

- Educate patients on the names and dosages of their regular medicines.

- Disseminate information widely using social media and local television channels.

\section{CONCLUSIONS}

We have presented our experience of successful delivery of essential healthcare services during the first nationwide lockdown which lasted for more than five weeks in our district. With a comprehensive contingency plan and proper planning at the local level, the services can be provided in a smooth and timely manner. Mobile clinics can be effectively utilized to provide majority of services during a lockdown with due attention to physical distancing and use of personal protective equipment ${ }^{7}$. While mitigating the impact of COVID-19 on routine healthcare, it is crucial to ensure continued access to essential healthcare services to the people ${ }^{8}$. We hope that our experience will prove useful to other districts and hospitals in planning and operationali- zing essential healthcare services to the public during future lockdowns.

\section{ACKNOWLEDMENTS}

We acknowledge the support of the administration and staff of CRRH, Gelephu who worked selflessly during the first nationwide lockdown.

\section{REFERENCES}

1. Tshokey T. An update on COVID-19 in Bhutan. Bhutan Health Journal [Internet]. 2020 [cited 9 July 2021];6(1):III. [Full Text | DOI]

2. Bhutan: WHO Coronavirus Disease (COVID-19) Dashboard With Vaccination Data [Internet]. covid19.who.int. 2021 [cited 9 August 2021]. [Full Text]

3. Haug N, Geyrhofer L, Londei A, Dervic E, Desvars-Larrive A, Loreto V, et al. Ranking the effectiveness of worldwide COVID-19 government interventions. Nature Human Behaviour. 2020;4(12):1303-1312. [Full Text | DOI]

4. Maintaining Essential Health Services During COVID-19 in Low Resource, Non-U.S. Settings [Internet]. Centers for Disease Control and Prevention. 2021 [cited 9 August 2021]. [Full Text]

5. Essential Health Packages: What are they for? What do they change? [Internet]. 4th ed. World Health Organization; 2021 [cited 9 August 2021]. [Full Text]

6. Essential Health Services During the Lockdown [Internet]. 2nd ed. Ministry of Health, Royal Government of Bhutan; 2021 [cited 9 August 2021]. [Full Text]

7. Enabling Delivery of Essential Health Services during the COVID 19 Outbreak: Guidance note [Internet]. Ministry of Health and Family Welfare, Government of India; 2020 [cited 9 August 2021]. [Full Text]

8. Blanchet K, Alwan A, Antoine C, Cros M, Feroz F, Amsalu Guracha $\mathrm{T}$ et al. Protecting essential health services in lowincome and middle-income countries and humanitarian settings while responding to the COVID-19 pandemic. BMJ Global Health. 2020;5(10):e003675. [Full Text | DOI] 